\title{
DESARROLLO DE UN SISTEMA DE CONTROL PARA LA CAPTURA Y MEDICIÓN EXPERIMENTAL DE LA EFICIENCIA Y CURVA CARACTERÍSTICA I-V EN TIEMPO REAL DE UN SISTEMA FOTOVOLTAICO UTILIZANDO LABVIEW® Y ARDUINO
}

Juan Pablo Vargas B. y Gonzalo Navia A.

\section{RESUMEN}

En el presente artículo se presenta un sistema de control para la visualización en tiempo real de datos experimentales de la curva de eficiencia y de la curva característica I-V (corriente versus voltaje) de un panel fotovoltaico (FV) SUNTECH-STP030-12. El sistema está compuesto de un programa desarrollado en LabView® y un circuito electrónico de captura y procesamiento de datos. Se utilizó un microprocesador Arduino UNO R3 para la interface entre los datos del panel FV y el programa en LabView®. Los resultados muestran que es posible utilizar microprocesadores de bajo costo con suficiente capacidad de procesamiento en tiempo real de la información obtenida de los paneles FV.

Palabras Clave: Paneles Fotovoltaicos, Arduino, LabView ${ }^{\circledR}$, Sistemas de Control. 